\title{
P-0537- Early life stress effects on pancreatic HB9 expression and insulin secretion in chronically stressed young adult rats
}

\author{
H. Zardooz ${ }^{1}$, F. Sadeghimahalli ${ }^{2}$, F. Khodagholi ${ }^{3}$, R. Karbaschi ${ }^{4}$, M. Sahraei ${ }^{5}$ \\ ${ }^{1}$ Neurophysiology Research Center, Department of Physiology, Shahid Beheshti University of \\ Medical Sciences, Tehran, Iran.
}

${ }^{2} E d u c a t i o n$ Development Center, Mazandaran University of Medical Sciences, Sari, Iran.

${ }^{3}$ Neuroscience Research Center, Shahid Beheshti University of Medical Sciences, Tehran, Iran.

${ }^{4}$ Faculty of Nursing and Midwifery, Shahid Beheshti University of Medical Sciences, Tehran, Iran. ${ }^{5}$ Faculty of Dentistry, Shahid Beheshti University of Medical Sciences, Tehran, Iran.
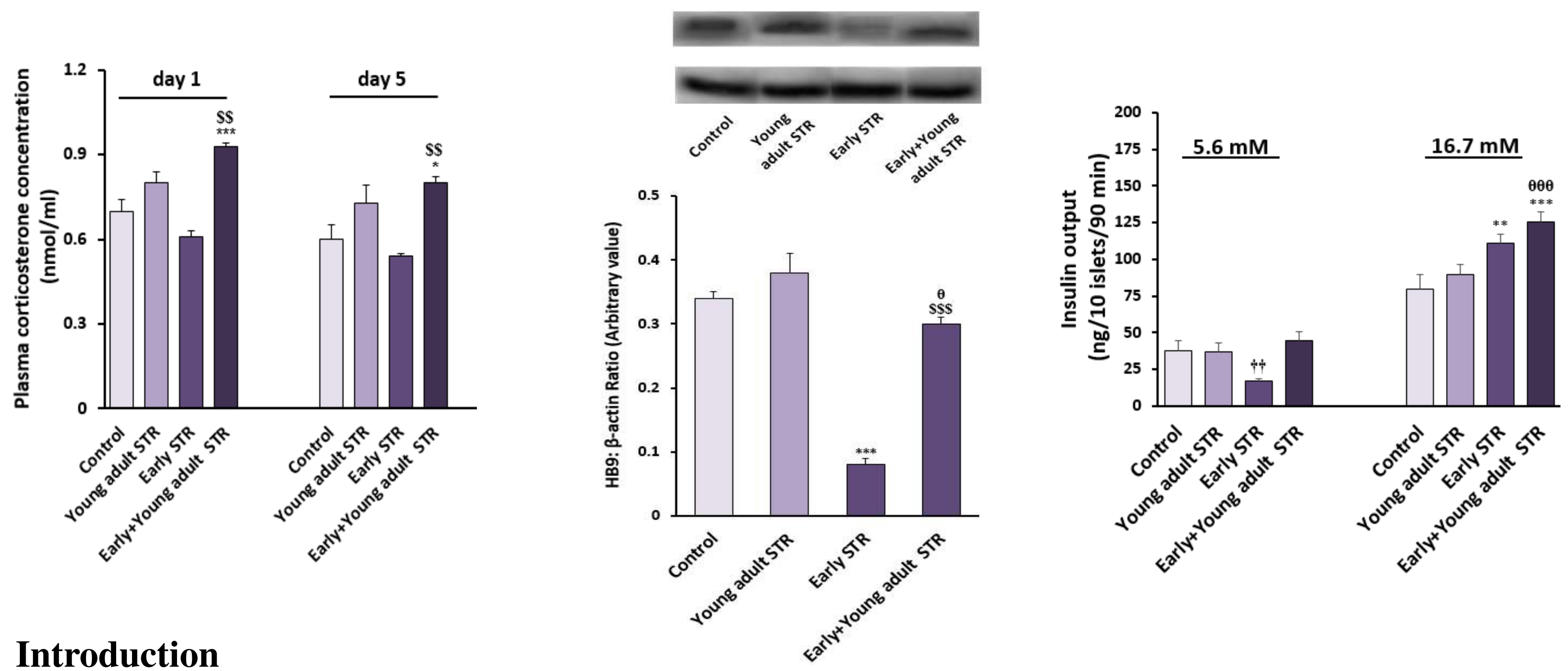

\section{Introduction}

Early postnatal exposure to stress may predispose neonates to certain disorders including glucose metabolism defects in later life. Pancreas is an immature organ at birth and HB9 as a transcription factor plays a role in the final development and differentiation of pancreatic beta cells. Also, studies have shown that stress could alter the expression of transcription factors. This study investigated the effect of early life stress on the expression of pancreatic HB9 protein and insulin secretion from isolated islets in young adult male Wistar rats subjected to chronic stress.

\section{Methods}

Male pups from different litters were randomly allocated into four different groups ( $\mathrm{n}=8 /$ group). The study groups consisted of the Control; Early STR; Young adult STR; and Early+Young adult STR. Foot shock stress was induced twice daily for 5 consecutive days by a communication box at two levels of age: early life at 2 weeks of age $(0.5 \mathrm{~mA}, 1 \mathrm{~Hz}$, lasting $10 \mathrm{~s}$, every $60 \mathrm{~s}$ for $30 \mathrm{~min})$; young adulthood at $8-10$ weeks of age $(1 \mathrm{~mA}, 1 \mathrm{~Hz}$, for $10 \mathrm{~s}$ every $60 \mathrm{~s}$ for $60 \mathrm{~min})$. After the last exposure to stress, isolated islets' insulin secretion, and pancreatic amounts of HB9 protein were measured. One- and Two-Way ANOVA were used for statistical analysis.

\section{Results}

In adulthood, plasma corticosterone concentration showed a significant increase only in Early+Young adult STR group compared to the control

\section{References}

1- Sadeghimahalli et al. Endocrine. 2015;48:493-503.

2- Maniam et al. Frontiers in Endocrinology. 2014;5:73.

3- Harrison et al. Nature Genetics. 1999; 23:71-5. and Early STR groups, immediately after the first and the last stress exposure. The amount of HB9 protein level was significantly lower in Early STR and Early+Young adult STR groups than the Control and Young adult STR groups respectively. However, it showed an increment in Early+Young adult STR group compared to the Early STR group. The insulin output from pancreatic isolated islets, in response to basal glucose concentration $(5.6 \mathrm{mM})$, was lower in Early STR group than the Control and Early+Young adult STR groups, whereas in response to high glucose concentration $(16.7 \mathrm{mM})$ showed a higher value than its respective Control group. In the Early+Young adult STR group the insulin output showed a higher value in response to $16.7 \mathrm{mM}$ glucose concentration but no difference in response to $5.6 \mathrm{mM}$ glucose compared to the Control and young adult STR groups.

\section{Conclusion}

Early life stress could alter hypothalamic-pituitary-adrenal axis activity in adulthood and therefore may affect plasma corticosterone level and HB9 protein expression level in the presence or absence of adulthood stress. Moreover, early life stress possibly by decreasing HB9 protein levels decreases insulin secretion ability of the isolated islets in response to basal glucose level and makes the organism susceptible to impaired glucose metabolism in adulthood. 